\title{
The effect of a protected mealtime policy on the energy intake and frequency of non-urgent interruptions during mealtimes at the Royal Bournemouth Hospital
}

\author{
C. M Stuckey, L. E. Bakewell and G. M. Ford \\ Nutrition and Dietetic Department, The Royal Bournemouth Hospital, Bournemouth BH7 7DW, UK
}

\begin{abstract}
Malnutrition is both a cause and consequence of disease effecting up to $30 \%$ of patients admitted to hospital ${ }^{(1)}$ and the public cost has been estimated at $£ 13$ billion a year ${ }^{(2)}$. The protected mealtime initiative (PMI), currently under the care of the national patient safety agency, aims to allow patients to eat their meals without unnecessary interruption and enable staff to assist those who are unable to eat independently ${ }^{(3)}$. Two years ago the PMI was introduced onto one ward at the Royal Bournemouth Hospital. Results of an audit showed a positive impact on the number of interruptions during the mealtime and the energy intake of patients ${ }^{(4)}$. Following this, protected mealtimes were introduced across the whole hospital. This audit, 6 months later, shows the effect on patients' mealtime experience and their energy intake.

Over a 2 week period all patients in one 6-bedded bay in each ward were observed at a lunchtime by a dietitian. The number and reason for all non-urgent interruptions was recorded. The menu choices of each patient and amount consumed was recorded and the energy content of the meal estimated using a standardised tool. Patients nil-by-mouth or on clear fluids only were excluded. The data were compared with data collected in the previous year before protected mealtimes was introduced.

Ninety seven patients were included in the baseline audit and 102 patients were included following the introduction of the PMI. At baseline, $45 \%$ of patients experienced one or more non-urgent interruptions during the mealtime. This decreased to $29 \%$ of patients after the PMI. The reason for interruptions can be seen below and a similar pattern was seen at baseline. The mean energy intake was $1907.904 \mathrm{~kJ} v .2125 .472 \mathrm{~kJ}(456 \mathrm{kcal} v .508 \mathrm{kcal})$ before and after PMI respectively. There was no difference in the intake of patients who were interrupted compared to those that were not.
\end{abstract}

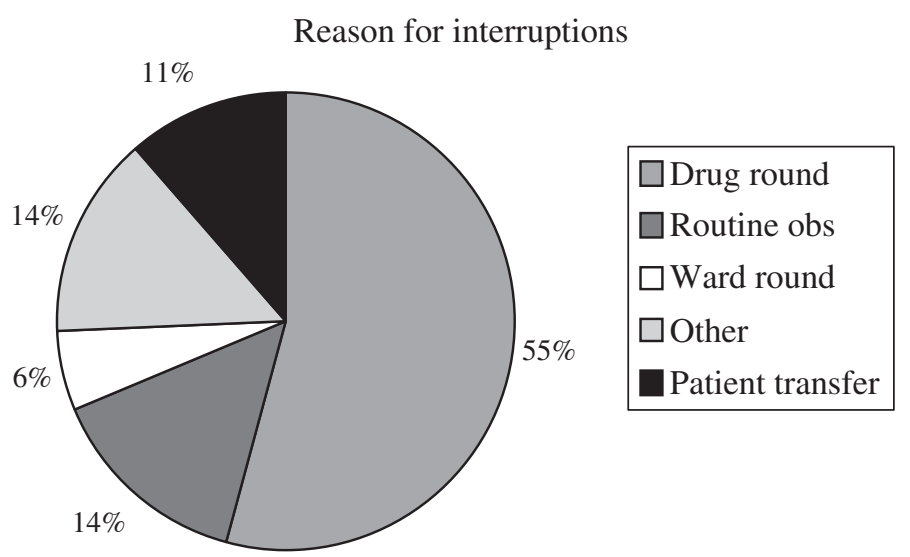

The introduction of the PMI at the Royal Bournemouth Hospital has decreased the number of non-urgent interruptions during meals and there is a trend towards an increased calorie intake. The patients' views of the PMI were not audited but the assumption is made that fewer interruptions leads to an improved experience. This could be audited in the future. Although an increase in the intake of patients at mealtimes would be beneficial to tackle malnutrition in hospitals, the aim of the national PMI is to improve patient experience not nutritional intake ${ }^{(3)}$. Our audit supports this function.

1. Russell CA \& Elia M (2009) Nutrition Screening Survey in the UK 2008: Hospitals, Care Homes and Mental Health Units. BAPEN.

2. Elia M \& Smith RM (2009) Improving nutritional care and treatment: perspectives and recommendations from population groups, patients and carers. BAPEN.

3. National Patient Safety (2009) Protected Mealtimes Review: Findings and Recommendations Report.

4. Stuckey C, O'Malley G, Matthias S et al. (2009) The introduction of protected mealtimes reduced interruptions and increases nutritional intake. Gut 58, Suppl. 1, A79-A80. 